Georgian Mathematical Journal

Volume 12 (2005), Number 4, 637-658

\title{
WAVELET FRAMES FOR DISTRIBUTIONS IN ANISOTROPIC BESOV SPACES
}

\author{
DOROTHEE D. HAROSKE AND ERIKA TAMÁSI
}

\begin{abstract}
This paper deals with wavelet frames in anisotropic Besov spaces $B_{p q}^{s, a}\left(\mathbb{R}^{n}\right), s \in \mathbb{R}, 0<p, q \leq \infty$, and $a=\left(a_{1}, \ldots, a_{n}\right)$ is an anisotropy, with $a_{i}>0, i=1, \ldots, n, a_{1}+\cdots+a_{n}=n$. We present sub-atomic and wavelet decompositions for a large class of distributions. To some extent our results can be regarded as anisotropic counterparts of those recently obtained in [38].
\end{abstract}

2000 Mathematics Subject Classification: 46E35, 42B35, 42C40.

Key words and phrases: Anisotropic function spaces, sub-atomic decomposition, wavelet decomposition, wavelet frames.

\section{INTRODUCTION}

Let $1<p<\infty$ and $\left(s_{1}, \ldots, s_{n}\right)$ be an $n$-tuple of natural numbers, then

$$
W_{p}^{s, a}\left(\mathbb{R}^{n}\right)=\left\{f \in \mathcal{S}^{\prime}\left(\mathbb{R}^{n}\right):\left\|f\left|L_{p}\left(\mathbb{R}^{n}\right)\left\|+\sum_{k=1}^{n}\right\| \frac{\partial^{s_{k}} f}{\partial x_{k}^{s_{k}}}\right| L_{p}\left(\mathbb{R}^{n}\right)\right\|<\infty\right\}
$$

is the classical anisotropic Sobolev space on $\mathbb{R}^{n}$. It is obvious that, unlike the case of the usual (isotropic) Sobolev space $\left(s_{1}=\cdots=s_{n}\right)$, the smoothness properties of an element from $W_{p}^{s, a}\left(\mathbb{R}^{n}\right)$ depend on a chosen direction in $\mathbb{R}^{n}$. The number $s$, defined by

$$
\frac{1}{s}=\frac{1}{n}\left(\frac{1}{s_{1}}+\cdots+\frac{1}{s_{n}}\right)
$$

is usually called the mean smoothness, and $a=\left(a_{1}, \ldots, a_{n}\right)$,

$$
a_{1}=\frac{s}{s_{1}}, \ldots, a_{n}=\frac{s}{s_{n}}
$$

characterizes the anisotropy. Similar to the isotropic situation, more general anisotropic Bessel potential spaces (fractional Sobolev spaces) $H_{p}^{s, a}\left(\mathbb{R}^{n}\right.$ ), where $1<p<\infty, s \in \mathbb{R}$ and $a=\left(a_{1}, \ldots, a_{n}\right)$ is a given anisotropy, fit in the scales of anisotropic Besov spaces $B_{p q}^{s, a}\left(\mathbb{R}^{n}\right)$ and anisotropic Triebel-Lizorkin spaces $F_{p q}^{s, a}\left(\mathbb{R}^{n}\right)$, respectively. The theory of anisotropic spaces was developed parallel to the theory for isotropic spaces; we refer in particular to the monographs [25] and [4] (and the papers referred to therein). It is well known that this theory is a more or less complete counterpart to the basic facts (definitions, description via differences and derivatives, elementary properties, embeddings for different metrics, interpolation) of isotropic spaces $B_{p q}^{s}\left(\mathbb{R}^{n}\right)$ and $F_{p q}^{s}\left(\mathbb{R}^{n}\right)$. We shall use the Fourier-analytical definition of $B_{p q}^{s, a}\left(\mathbb{R}^{n}\right), F_{p q}^{s, a}\left(\mathbb{R}^{n}\right)$, where any function $f \in$ 
$\mathcal{S}^{\prime}\left(\mathbb{R}^{n}\right)$ is decomposed in a sum of entire analytic functions $\left(\varphi_{j} \widehat{f}\right)^{\vee}$ and this decomposition, measured in $\ell_{q}$ and $L_{p}\left(\mathbb{R}^{n}\right)$, respectively, is used to introduce these spaces. This concept goes back to [32] and [31], see [27, Chapter 4]. The theory of isotropic spaces was studied systematically in [33], [34], [35], and, more recently, in [36].

In the theory of isotropic function spaces it has turned out that other wellknown types of decompositions in simpler building blocks like atoms, quarks or wavelets, are better adapted to some problems and applications connected with the study of function spaces. In contrast to sub-atomic decompositions (based on the so-called quarks) introduced not long ago in [35], see also [36], atomic decompositions have a history of some twenty years and a historical report on this topic is given in $[34,1.9]$, cf. also [1]. We only want to recall that (smooth) atoms in isotropic spaces $B_{p q}^{s}\left(\mathbb{R}^{n}\right)$ and $F_{p q}^{s}\left(\mathbb{R}^{n}\right)$ as defined in [14], [15] (cf. also [16]) proved to be a powerful tool in the theory of function spaces.

In the last years several authors have been concerned with the problem of obtaining useful decompositions of anisotropic function spaces, too. A construction of unconditional bases in spaces $B_{p q}^{s, a}\left(\mathbb{R}^{n}\right)$ and $F_{p q}^{s, a}\left(\mathbb{R}^{n}\right)$ using Meyer wavelets was obtained in [2], [3]; see, more recent works [18], [17], [19]; a different approach, involving the $\varphi$-transform of Frazier and Jawerth (see [15], [16]) was developed in [9], [10], see also [28]. The most recent contributions we know are made in [7], [21], [22]; see also [5], [6].

Our main aim in the present paper is to prove an anisotropic counterpart of some recent results on wavelet frames parallel to the isotropic case in [38]. Essential ingredients in our approach are representations by local means, atomic and sub-atomic decompositions according to [13], [12]; see also [8], [41], [42]. More precisely, let $a=\left(a_{1}, \ldots, a_{n}\right)$ be a given anisotropy, $|\cdot|_{a}$ an anisotropic distance, and $k$ be a non-negative $C^{\infty}$-function in $\mathbb{R}^{n}$ such that $\operatorname{supp} k \subset\{y \in$ $\left.\mathbb{R}^{n}:|y|_{a}<2^{J}, y_{j}>0, j=1, \ldots, n\right\}$ for some $J \in \mathbb{N}$, with

$$
\sum_{m \in \mathbb{Z}^{n}} k(x-m)=1, \quad x \in \mathbb{R}^{n} .
$$

For $\beta \in \mathbb{N}_{0}^{n}$ and $x \in \mathbb{R}^{n}$, put $k^{\beta}(x)=\left(2^{-J a} x\right)^{\beta} k(x)$, and let for $f \in \mathcal{S}^{\prime}\left(\mathbb{R}^{n}\right)$,

$$
k^{\beta}(t, f)(x)=\int_{\mathbb{R}^{n}} k^{\beta}(y) f\left(x+t^{a} y\right) \mathrm{d} y, \quad t>0, \quad x \in \mathbb{R}^{n},
$$

be the corresponding (anisotropic) local means (see [34, 2.4.6/1] for the isotropic version and [13] for its anisotropic counterpart). Let $\omega \in \mathcal{S}\left(\mathbb{R}^{n}\right)$ with $\operatorname{supp} \omega \subset$ $(-\pi, \pi)^{n}, \omega(x)=1$ if $|x|_{a} \leq 2$. Parallel to the isotropic case we introduce

$$
\omega^{\beta}(x)=\frac{i^{|\beta|} 2^{J a \beta}}{(2 \pi)^{n} \beta !} x^{\beta} \omega(x) \quad \text { and } \quad \Omega^{\beta}(x)=\sum_{m \in \mathbb{Z}^{n}}\left(\omega^{\beta}\right)^{\vee}(m) e^{-i m x},
$$

where $x \in \mathbb{R}^{n}, \beta \in \mathbb{N}_{0}^{n}$. Assume that $\varphi_{0}$ is some $C^{\infty}$-function on $\mathbb{R}^{n}$ with $\varphi_{0}(x)=$ 1 if $|x|_{a} \leq 1$, and $\varphi_{0}(x)=0$ if $|x|_{a} \geq \frac{3}{2}$; put $\varphi(x)=\varphi_{0}(x)-\varphi_{0}\left(2^{a} x\right)$. For $\beta \in \mathbb{N}_{0}^{n}$ we introduce (anisotropic) father wavelets $\Phi_{F}^{\beta}(x)$ and mother wavelets 
$\Phi_{M}^{\beta}(x)$ by

$$
\left(\Phi_{F}^{\beta}\right)^{\vee}(\xi)=\varphi_{0}(\xi) \Omega^{\beta}(\xi), \quad\left(\Phi_{M}^{\beta}\right)^{\vee}(\xi)=\varphi(\xi) \Omega^{\beta}(\xi), \quad \xi \in \mathbb{R}^{n},
$$

and use the abbreviation

$$
\Phi_{j m}^{\beta}(x)= \begin{cases}\Phi_{F}^{\beta}(x-m), & \text { if } j=0, \\ \Phi_{M}^{\beta}\left(2^{j a} x-m\right), & \text { if } j \in \mathbb{N},\end{cases}
$$

$\beta \in \mathbb{N}_{0}^{n}, j \in \mathbb{N}_{0}, m \in \mathbb{Z}^{n}$. Our first main result now establishes a quarkonial (sub-atomic) decomposition in the sense that for given $0<p \leq \infty$, $s>n \max \left(\frac{1}{p}-1,0\right), \varrho \geq 0$, and an anisotropy $a, f \in \mathcal{S}^{\prime}\left(\mathbb{R}^{n}\right)$ is an element of $B_{p p}^{s, a}\left(\mathbb{R}^{n}\right)$ if and only if it can be represented as

$$
f=\sum_{\beta \in \mathbb{N}_{0}^{n}} \sum_{j=0}^{\infty} \sum_{m \in \mathbb{Z}^{n}} \lambda_{j m}^{\beta} k^{\beta}\left(2^{j a} x-m\right), \quad x \in \mathbb{R}^{n},
$$

the series being absolutely convergent in $L_{\max (p, 1)}\left(\mathbb{R}^{n}\right)$, with

$$
\left(\sum_{\beta \in \mathbb{N}_{0}^{n}} \sum_{j=0}^{\infty} \sum_{m \in \mathbb{Z}^{n}} 2^{\varrho a \beta p+j(s-n / p) p}\left|\lambda_{j m}^{\beta}\right|^{p}\right)^{1 / p}<\infty .
$$

Moreover, for $f \in B_{p p}^{s, a}\left(\mathbb{R}^{n}\right)$, (0.6) with $\lambda_{j m}^{\beta}$ replaced by $\lambda_{j m}^{\beta}(f)=2^{j n}\left(f, \Phi_{j m}^{\beta}\right)$, $\beta \in \mathbb{N}_{0}^{n}, j \in \mathbb{N}_{0}, m \in \mathbb{Z}^{n}$, is an optimal representation, i.e.,

$$
\left\|f \mid B_{p p}^{s, a}\left(\mathbb{R}^{n}\right)\right\| \sim\left(\sum_{\beta \in \mathbb{N}_{0}^{n}} \sum_{j=0}^{\infty} \sum_{m \in \mathbb{Z}^{n}} 2^{\varrho a \beta p+j(s-n / p) p}\left|\lambda_{j m}^{\beta}(f)\right|^{p}\right)^{1 / p} .
$$

In the case of negative $s$ we have a parallel "dual" result, namely that for $1<p \leq \infty, s<0$, and a given anisotropy $a, f \in \mathcal{S}^{\prime}\left(\mathbb{R}^{n}\right)$ is an element of $B_{p p}^{s, a}\left(\mathbb{R}^{n}\right)$ if and only if it can be represented as

$$
f=\sum_{\beta \in \mathbb{N}_{0}^{n}} \sum_{j=0}^{\infty} \sum_{m \in \mathbb{Z}^{n}} \lambda_{j m}^{\beta} \Phi_{j m}^{\beta}, \quad x \in \mathbb{R}^{n},
$$

the series being unconditionally convergent in $\mathcal{S}^{\prime}\left(\mathbb{R}^{n}\right)$, with

$$
\left(\sum_{\beta \in \mathbb{N}_{0}^{n}} \sum_{j=0}^{\infty} \sum_{m \in \mathbb{Z}^{n}} 2^{j(s-n / p) p}\left|\lambda_{j m}^{\beta}\right|^{p}\right)^{1 / p}<\infty .
$$

Again, we have an optimal representation for $f \in B_{p p}^{s, a}\left(\mathbb{R}^{n}\right)$ if in (0.7) we replace the coefficients $\lambda_{j m}^{\beta}$ by $k_{j m}^{\beta}(f)=k^{\beta}\left(2^{-j}, f\right)\left(2^{-j a} m\right), j \in \mathbb{N}_{0}, m \in \mathbb{Z}^{n}, \beta \in \mathbb{N}_{0}^{n}$, see $(0.4)$.

The paper is organized as follows. In Section 1 we collect fundamentals about anisotropic Besov spaces and recall the atomic decomposition in such spaces. Our main results are presented in Section 2, whereas all the proofs (and some auxiliary assertions) are given in Section 3. 


\section{Preliminaries}

1.1. General notation. As usual, $\mathbb{R}^{n}$ denotes the $n$-dimensional real Euclidean space, $\mathbb{N}$ the collection of all natural numbers, $\mathbb{N}_{0}=\mathbb{N} \cup\{0\}, \mathbb{C}$ stands for complex numbers, and $\mathbb{Z}^{n}$ means the lattice of all points in $\mathbb{R}^{n}$ with integer-valued components. We use the equivalence " $\sim$ " in $\varphi(x) \sim \psi(x)$ always to mean that there are two positive numbers $c_{1}$ and $c_{2}$ such that

$$
c_{1} \varphi(x) \leq \psi(x) \leq c_{2} \varphi(x)
$$

for all admitted values of $x$, where $\varphi, \psi$ are non-negative functions. If $a \in \mathbb{R}$, then $a_{+}:=\max (a, 0)$. Let $\alpha=\left(\alpha_{1}, \ldots, \alpha_{n}\right) \in \mathbb{N}_{0}^{n}$ be a multi-index, then

$$
|\alpha|=\alpha_{1}+\cdots+\alpha_{n}, \quad \alpha !=\alpha_{1} ! \cdots \alpha_{n} !, \quad \alpha \in \mathbb{N}_{0}^{n}
$$

the derivatives $D^{\alpha}$ have the usual meaning, $x^{\alpha}$ means $x^{\alpha}=x_{1}^{\alpha_{1}} \cdots x_{n}^{\alpha_{n}}$ for $x=\left(x_{1}, \ldots, x_{n}\right) \in \mathbb{R}^{n}$, and $\alpha \gamma=\alpha_{1} \gamma_{1}+\cdots+\alpha_{n} \gamma_{n}, \gamma \in \mathbb{R}^{n}$, stands for the scalar product in $\mathbb{R}^{n}$.

Given two quasi-Banach spaces $X$ and $Y$, we write $X \hookrightarrow Y$ if $X \subset Y$ and the natural embedding of $X$ in $Y$ is continuous. All unimportant positive constants will be denoted by $c$, occasionally with additional subscripts within the same formula. We shall always deal with function spaces on $\mathbb{R}^{n}$; so for convenience we shall usually omit the " $\mathbb{R}^{n}$ " in their notation.

1.2. Anisotropic function spaces. Let $a=\left(a_{1}, \ldots, a_{n}\right)$ be a fixed $n$-tuple of positive numbers with $a_{1}+\cdots+a_{n}=n$, then we call $a$ an anisotropy. We denote $a_{\min }=\min \left\{a_{i}: 1 \leq i \leq n\right\}$ and $a_{\max }=\max \left\{a_{i}: 1 \leq i \leq n\right\}$. If $a=(1, \ldots, 1)$ we speak about the "isotropic case".

The action of $t \in[0, \infty)$ on $x \in \mathbb{R}^{n}$ is defined by the formula

$$
t^{a} x=\left(t^{a_{1}} x_{1}, \ldots, t^{a_{n}} x_{n}\right) .
$$

For $t>0$ and $s \in \mathbb{R}$ we put $t^{s a} x=\left(t^{s}\right)^{a} x$. In particular we write $t^{-a} x=\left(t^{-1}\right)^{a} x$ and $2^{-j a} x=\left(2^{-j}\right)^{a} x$.

Definition 1.1. An anisotropic distance function is a continuous function $u: \mathbb{R}^{n} \rightarrow \mathbb{R}$ with the properties $u(x)>0$ if $x \neq 0$ and $u\left(t^{a} x\right)=t u(x)$ for all $t>0$ and all $x \in \mathbb{R}^{n}$.

Remark 1.2. It is easy to see that $u_{\lambda}: \mathbb{R}^{n} \rightarrow \mathbb{R}$ defined by

$$
u_{\lambda}(x)=\left(\sum_{i=1}^{n}\left|x_{i}\right|^{\frac{\lambda}{a_{i}}}\right)^{1 / \lambda}
$$

is an anisotropic distance function for every $0<\lambda<\infty, u_{2}$ is usually called an anisotropic distance from $x$ to the origin, see $[27,4.2 .1]$. It is well known, see $[10,1.2 .3]$ and $[41,1.4]$, that any two anisotropic distance functions $u$ and $u^{\prime}$ are equivalent (in the sense that there exist constants $c, c^{\prime}>0$ such that $c u(x) \leq$ $u^{\prime}(x) \leq c^{\prime} u(x)$ for all $\left.x \in \mathbb{R}^{n}\right)$ and that if $u$ is an anisotropic distance function there exists a constant $c>0$ such that $u(x+y) \leq c(u(x)+u(y))$ for all $x, y \in \mathbb{R}^{n}$. We are interested in using smooth anisotropic distance functions. Note that for appropriate values of $\lambda$ one can obtain arbitrary (finite) smoothness of the 
function $u_{\lambda}$ from $(1.3)$, cf. $[10,1.2 .4]$. A standard method concerning the construction of anisotropic distance functions in $C^{\infty}\left(\mathbb{R}^{n} \backslash\{0\}\right)$ is given in [30].

For $x=\left(x_{1}, \ldots, x_{n}\right) \in \mathbb{R}^{n}, x \neq 0$, let $|x|_{a}$ be a unique positive number $t$ such that

$$
\frac{x_{1}^{2}}{t^{2 a_{1}}}+\cdots+\frac{x_{n}^{2}}{t^{2 a_{n}}}=1
$$

and let $|0|_{a}=0$; then $|\cdot|_{a}$ is an anisotropic distance function in $C^{\infty}\left(\mathbb{R}^{n} \backslash\{0\}\right)$, see $[41,1.4 / 3,8]$. Plainly, in the isotropic case $|x|_{a}$ is the Euclidean distance from $x$ to the origin.

Before introducing the function spaces to be considered we need to recall some notation. By $\mathcal{S}$ we denote the Schwartz space of all complex-valued, infinitely differentiable and rapidly decreasing functions on $\mathbb{R}^{n}$ and by $\mathcal{S}^{\prime}$ the dual space of all tempered distributions on $\mathbb{R}^{n}$. Furthermore, $L_{p}$ with $0<p \leq \infty$, stands for the usual quasi-Banach space with respect to the Lebesgue measure quasinormed by

$$
\left\|f \mid L_{p}\right\|:=\left(\int_{\mathbb{R}^{n}}|f(x)|^{p} \mathrm{~d} x\right)^{1 / p}
$$

with the obvious modification if $p=\infty$. If $\varphi \in \mathcal{S}$, then

$$
\widehat{\varphi}(\xi) \equiv(\mathcal{F} \varphi)(\xi):=(2 \pi)^{-n / 2} \int_{\mathbb{R}^{n}} e^{-i x \xi} \varphi(x) \mathrm{d} x, \quad x \in \mathbb{R}^{n},
$$

denotes the Fourier transform of $\varphi$. As usual, $\mathcal{F}^{-1} \varphi$ or $\varphi^{\vee}$ stands for the inverse Fourier transform given by the right-hand side of (1.5) with $i$ in place of $-i$. Here $x \xi$ denotes the scalar product in $\mathbb{R}^{n}$. Both $\mathcal{F}$ and $\mathcal{F}^{-1}$ are extended to $\mathcal{S}^{\prime}$ in standard manner. Let $\varphi_{0} \in \mathcal{S}$ be such that

$$
\varphi_{0}(x)=1 \quad \text { if } \quad|x|_{a} \leq 1 \quad \text { and } \operatorname{supp} \varphi_{0} \subset\left\{x \in \mathbb{R}^{n}:|x|_{a} \leq 2\right\},
$$

and for each $j \in \mathbb{N}$ let

$$
\varphi_{j}^{a}(x):=\varphi_{0}\left(2^{-j a} x\right)-\varphi_{0}\left(2^{(-j+1) a} x\right), \quad x \in \mathbb{R}^{n} .
$$

Then the sequence $\left(\varphi_{j}^{a}\right)_{j=0}^{\infty}$ forms a smooth anisotropic dyadic resolution of unity, cf. [27, 4.2]. Let $f \in \mathcal{S}^{\prime}$, then the compact support of $\varphi_{j}^{a} \widehat{f}$ implies by the Paley-Wiener-Schwartz theorem that $\left(\varphi_{j}^{a} \widehat{f}\right)^{\vee}$ is an entire analytic function on $\mathbb{R}^{n}$.

Definition 1.3. Assume $0<p \leq \infty, 0<q \leq \infty, s \in \mathbb{R}, a$ be an anisotropy, and $\left(\varphi_{j}^{a}\right)_{j=0}^{\infty}$ a smooth anisotropic dyadic resolution of unity. Then

$$
B_{p q}^{s, a}=\left\{f \in \mathcal{S}^{\prime}:\left\|f \mid B_{p q}^{s, a}\right\|_{\varphi}=\left(\sum_{j=0}^{\infty} 2^{j s q}\left\|\left(\varphi_{j}^{a} \widehat{f}\right)^{\vee} \mid L_{p}\right\|^{q}\right)^{1 / q}<\infty\right\}
$$

(with the usual modification if $q=\infty$ ). 
Note that there is a parallel definition for spaces of type $F_{p q}^{s, a}, 0<p<\infty$, $0<q \leq \infty, s \in \mathbb{R}, a$ an anisotropy, when interchanging the order of $\ell_{q^{-}}$and $L_{p^{-}}$quasi-norms in (1.8). It is obvious, that the quasi-norm (1.8) depends on the chosen system $\left(\varphi_{j}^{a}\right)_{j \in \mathbb{N}_{0}}$, but not the space $B_{p q}^{s, a}$ (in the sense of equivalent quasi-norms); therefore in the sequel we omit the subscript $\varphi$ in our notation. It is well known that $B_{p q}^{s, a}$ are quasi-Banach spaces (Banach spaces if $p \geq 1$ and $q \geq 1$ ), and, as in the isotropic case, $\mathcal{S} \hookrightarrow B_{p q}^{s, a} \hookrightarrow \mathcal{S}^{\prime}$ for all admissible values of $p, q, s$, see $[33,2.3 .3]$. If $s \in \mathbb{R}$ and $0<p<\infty, 0<q<\infty$, then $\mathcal{S}$ is dense in $B_{p q}^{s, a}$, see $[41,3.5]$ and $[10,1.2 .10]$. Note that we have indicated the only (formal) difference from the isotropic counterparts of (1.8) by the additional superscript at the smooth anisotropic dyadic resolution of unity $\left(\varphi_{j}^{a}\right)_{j=0}^{\infty}$.

Remark 1.4. A systematic treatment of the theory of (isotropic) $B_{p q}^{s}$ (and $\left.F_{p q}^{s}\right)$ spaces can be found in the monographs [33], [34], [35] and [36]; see also [11] and [26]. A survey on the basic results for the (anisotropic) spaces $B_{p q}^{s, a}$ (and $\left.F_{p q}^{s, a}\right)$ is given in $[27,4.2 .1-4.2 .4]$ and $[20,2.1-2.2]$. In addition to the literature mentioned in our introduction, in the sequel we essentially rely on [13] and [12].

For convenience, in the case of $p=q$ we shall stick to the notation

$$
B_{p}^{s, a}=B_{p p}^{s, a} \quad \text { where } \quad 0<p \leq \infty, \quad s \in \mathbb{R}
$$

in the sequel.

To prove our main theorems, we need the following proposition, which is interesting in itself; thus we state it here separately.

Proposition 1.5. Let $0<p \leq \infty, s \in \mathbb{R}$, a be an anisotropy, and $\left(\varphi_{j}^{a}\right)_{j=0}^{\infty} a$ smooth anisotropic dyadic resolution of unity. Then for each $f \in B_{p}^{s, a}$,

$$
\left(\sum_{j=0}^{\infty} \sum_{m \in \mathbb{Z}^{n}} 2^{j(s-n / p) p}\left|\left(\varphi_{j}^{a} \widehat{f}\right)^{\vee}\left(2^{-j a} m\right)\right|^{p}\right)^{1 / p} \sim\left\|f \mid B_{p}^{s, a}\right\|
$$

(with the usual modification if $p=\infty$ ), where the equivalence constants are independent of $s$ and $f$.

1.3. Decomposition of anisotropic function spaces. Our main results formulated in Section 2 concern sub-atomic and wavelet representations of anisotropic spaces. The arguments given there are essentially based on the (known) corresponding atomic decompositions. Thus we recall some basic facts about anisotropic atoms and mainly rely on [13] in that context; see also [12].

Let $a=\left(a_{1}, \ldots, a_{n}\right), \nu \in \mathbb{N}_{0}$, and $m=\left(m_{1}, \ldots, m_{n}\right) \in \mathbb{Z}^{n}$, then we denote by $R_{\nu m}^{a}$ the rectangle in $\mathbb{R}^{n}$ centered at $2^{-\nu a} m=\left(2^{-\nu a_{1}} m_{1}, \ldots, 2^{-\nu a_{n}} m_{n}\right)$ which has the sides parallel to the axes and side lengths $2^{-\nu a_{1}}, \ldots, 2^{-\nu a_{n}}$, respectively. Note that $R_{0 m}^{a}$ is the cube with side length 1 . If $R_{\nu m}^{a}$ is such a rectangle in $\mathbb{R}^{n}$ and $c>0$, then $c R_{\nu m}^{a}$ is the rectangle in $\mathbb{R}^{n}$ concentric with $R_{\nu m}^{a}$ and with side lengths $c 2^{-\nu a_{1}}, \ldots, c 2^{-\nu a_{n}}$, respectively. 


\section{Definition 1.6.}

(i) Let $K \in \mathbb{R}, c>1$. A function $\varrho: \mathbb{R}^{n} \rightarrow \mathbb{C}$ for which there exist all derivatives $D^{\gamma} \varrho$ if $a \gamma \leq K$ (continuous if $K \leq 0$ ) is called an anisotropic $1_{K}$-atom if

$$
\begin{gathered}
\operatorname{supp} \varrho \subset c R_{0 m}^{a} \quad \text { for some } m \in \mathbb{Z}^{n}, \\
\left|D^{\gamma} \varrho(x)\right| \leq 1 \quad \text { if } \quad a \gamma \leq K, \quad \gamma \in \mathbb{N}_{0}^{n}, x \in \mathbb{R}^{n} .
\end{gathered}
$$

(ii) Let $s \in \mathbb{R}, 0<p \leq \infty, K, L \in \mathbb{R}$. A function $\varrho: \mathbb{R}^{n} \rightarrow \mathbb{C}$ for which there exist all derivatives $D^{\gamma} \varrho$ if $a \gamma \leq K$ (continuous if $K \leq 0$ ) is called an anisotropic $(s, p)_{K, L}$-atom if

$$
\begin{gathered}
\operatorname{supp} \varrho \subset c R_{\nu m}^{a} \quad \text { for some } \quad \nu \in \mathbb{N}, m \in \mathbb{Z}^{n}, \\
\left|D^{\gamma} \varrho(x)\right| \leq 2^{-\nu\left(s-\frac{n}{p}\right)+\nu a \gamma} \quad \text { if } \quad a \gamma \leq K, \quad \gamma \in \mathbb{N}_{0}^{n}, \quad x \in \mathbb{R}^{n}, \\
\int_{\mathbb{R}^{n}} x^{\beta} \varrho(x) \mathrm{d} x=0 \quad \text { if } a \beta \leq L, \quad \beta \in \mathbb{N}_{0}^{n} .
\end{gathered}
$$

If the atom $\varrho$ is located at $R_{\nu m}^{a}$ (that means $\operatorname{supp} \varrho \subset c R_{\nu m}^{a}$ with $\nu \in \mathbb{N}_{0}$, $\left.m \in \mathbb{Z}^{n}, c>1\right)$, then we denote it by $\varrho_{\nu m}^{a}$.

Remark 1.7. The value of $c>1$ in (1.11) and (1.13) is unimportant; it only indicates that at level $\nu$ some controlled overlapping of the supports of $\varrho_{\nu m}^{a}$ has to be allowed. The moment conditions (1.15) can be rewritten as

$$
D^{\beta} \widehat{\varrho}(0)=0 \text { if } a \beta \leq L,
$$

which shows that a sufficiently strong decay of $\widehat{\varrho}$ at the origin is required. If $L<0$ then (1.15) should be interpreted in the sense that there are no moment conditions. The normalizing factors in (1.12), (1.14) imply that there exists a constant $c>0$ such that for all these atoms we have $\left\|\varrho \mid B_{p q}^{s, a}\right\| \leq c$, see Theorem 1.9 below. Hence, as in the isotropic case, atoms are normalized building blocks satisfying certain moment conditions.

This construction generalizes isotropic atoms (cf. [14], [15] and the survey [16]). It is also slightly related to the concept of anisotropic building blocks (compactly supported and satisfying some norming and some moment conditions) used in [29] to define anisotropic Hardy spaces and to study the relation of these spaces to anisotropic Lipschitz and Campanato-Morrey spaces. As already mentioned, we use the presentation from [13], which itself was motivated by the isotropic counterparts in [34], [35].

Suitable anisotropic sequence spaces can be introduced as follows.

Definition 1.8. Let $0<p \leq \infty, 0<q \leq \infty$, and $a$ be an anisotropy. Then $b_{p q}$ is the collection of all sequences $\lambda=\left\{\lambda_{\nu m} \in \mathbb{C}: \nu \in \mathbb{N}_{0}, m \in \mathbb{Z}^{n}\right\}$ such that

$$
\left\|\lambda \mid b_{p q}\right\|=\left(\sum_{\nu=0}^{\infty}\left(\sum_{m \in \mathbb{Z}^{n}}\left|\lambda_{\nu m}\right|^{p}\right)^{q / p}\right)^{1 / q}
$$

(with the usual modification if $p=\infty$ and/or $q=\infty$ ) is finite. 
Again, note that there is a counterpart for spaces of type $F_{p q}^{s, a}$; the corresponding sequence spaces $f_{p q}^{a}$ can be introduced similarly, but we do not need them in the sequel. One can easily check that $b_{p q}$ are quasi-Banach spaces. For $0<p \leq \infty$ we introduce the abbreviation

$$
\sigma_{p}=n\left(\frac{1}{p}-1\right)_{+} .
$$

Theorem 1.9. Let $0<p \leq \infty, 0<q \leq \infty, s \in \mathbb{R}$ and let $K, L \in \mathbb{R}$ be such that

$$
\begin{gathered}
K \geq a_{\max }+s \quad \text { if } s \geq 0, \\
L \geq \sigma_{p}-s .
\end{gathered}
$$

Then $g \in \mathcal{S}^{\prime}$ belongs to $B_{p q}^{s, a}$ if and only if it can be represented as

$$
g=\sum_{\nu=0}^{\infty} \sum_{m \in \mathbb{Z}^{n}} \lambda_{\nu m} \varrho_{\nu m}^{a},
$$

the series being unconditionally convergent in $\mathcal{S}^{\prime}$, where $\varrho_{\nu m}^{a}$ are anisotropic $1_{K}$-atoms $(\nu=0)$ or anisotropic $(s, p)_{K, L}$-atoms $(\nu \in \mathbb{N})$ and $\lambda \in b_{p q}$ with $\lambda=\left\{\lambda_{\nu m}: \nu \in \mathbb{N}_{0}, m \in \mathbb{Z}^{n}\right\}$. Moreover

$$
\inf \left\|\lambda \mid b_{p q}\right\|,
$$

where the infimum is taken over all admissible representations (1.20), is an equivalent quasi-norm in $B_{p q}^{s, a}$.

Remark 1.10. The proof of this theorem - and of its counterpart for spaces $F_{p q}^{s, a}$ - is given in [13, Section 5.1]. The convergence in $\mathcal{S}^{\prime}$ can be obtained as a by-product of the proof using the same method as for its isotropic counterpart in $[35,13.9]$. As already mentioned, it generalizes atomic decomposition results in [14], [15], [35] to anisotropic function spaces.

Our main object is to study the anisotropic counterpart of the results from [38]; hence we closely follow that presentation, adapting it to our context when necessary, and keeping similar notation if possible. Let

$$
\mathbb{R}_{++}^{n}=\left\{y \in \mathbb{R}^{n}: y=\left(y_{1}, \ldots, y_{n}\right), y_{j}>0 \text { for all } j\right\}
$$

and let $k$ be a non-negative $C^{\infty}$-function in $\mathbb{R}^{n}$ with

$$
\operatorname{supp} k \subset\left\{y \in \mathbb{R}^{n}:|y|_{a}<2^{J}\right\} \cap \mathbb{R}_{++}^{n},
$$

for some $J \in \mathbb{N}$, and

$$
\sum_{m \in \mathbb{Z}^{n}} k(x-m)=1, \quad x \in \mathbb{R}^{n} .
$$

Recall that $x^{\beta}=x_{1}^{\beta_{1}} \cdots x_{n}^{\beta_{n}}$ where $x=\left(x_{1}, \ldots, x_{n}\right) \in \mathbb{R}^{n}$ and $\beta \in \mathbb{N}_{0}^{n}$, and put

$$
k^{\beta}(x)=\left(2^{-J a} x\right)^{\beta} k(x) \geq 0, \quad x \in \mathbb{R}^{n}, \beta \in \mathbb{N}_{0}^{n} .
$$

Let

$$
\lambda=\left\{\lambda_{j m}^{\beta} \in \mathbb{C}: j \in \mathbb{N}_{0}, m \in \mathbb{Z}^{n}, \beta \in \mathbb{N}_{0}^{n}\right\}
$$


For $s \in \mathbb{R}, 0<p \leq \infty$, and $\varrho \geq 0$, we introduce $b_{p}^{s, \varrho}$ by

$$
\left\|\lambda \mid b_{p}^{s, \varrho}\right\|=\left(\sum_{\beta \in \mathbb{N}_{0}^{n}} \sum_{j=0}^{\infty} \sum_{m \in \mathbb{Z}^{n}} 2^{\varrho a \beta p+j(s-n / p) p}\left|\lambda_{j m}^{\beta}\right|^{p}\right)^{1 / p} .
$$

Let

$$
\omega \in \mathcal{S}, \operatorname{supp} \omega \subset(-\pi, \pi)^{n}, \omega(x)=1 \quad \text { if } \quad|x|_{a} \leq 2,
$$

and, taking into account (1.1), let

$$
\omega^{\beta}(x)=\frac{i^{|\beta|} 2^{J a \beta}}{(2 \pi)^{n} \beta !} x^{\beta} \omega(x) \quad \text { for } x \in \mathbb{R}^{n}, \quad \beta \in \mathbb{N}_{0}^{n} .
$$

Finally, let

$$
\Omega^{\beta}(x)=\sum_{m \in \mathbb{Z}^{n}}\left(\omega^{\beta}\right)^{\vee}(m) e^{-i m x}, \quad x \in \mathbb{R}^{n}, \quad \beta \in \mathbb{N}_{0}^{n} .
$$

Definition 1.11. Let $\varphi_{0}$ be a $C^{\infty}$-function in $\mathbb{R}^{n}$ with

$$
\varphi_{0}(x)=1 \quad \text { if } \quad|x|_{a} \leq 1, \quad \text { and } \quad \varphi_{0}(x)=0 \quad \text { if } \quad|x|_{a} \geq \frac{3}{2}
$$

and let $\varphi(x)=\varphi_{0}(x)-\varphi_{0}\left(2^{a} x\right)$ and $\beta \in \mathbb{N}_{0}^{n}$. Father wavelets $\Phi_{F}^{\beta}(x)$ and mother wavelets $\Phi_{M}^{\beta}(x)$ are given by

$$
\begin{aligned}
& \left(\Phi_{F}^{\beta}\right)^{\vee}(\xi)=\varphi_{0}(\xi) \Omega^{\beta}(\xi), \quad \xi \in \mathbb{R}^{n}, \\
& \left(\Phi_{M}^{\beta}\right)^{\vee}(\xi)=\varphi(\xi) \Omega^{\beta}(\xi), \quad \xi \in \mathbb{R}^{n} .
\end{aligned}
$$

Remark 1.12. Our assumption $\omega^{\beta} \in \mathcal{S}$ implies that $\left(\Phi_{F}^{\beta}\right)^{\vee},\left(\Phi_{M}^{\beta}\right)^{\vee}$, and hence $\Phi_{F}^{\beta}, \Phi_{M}^{\beta}$, too, are elements of $\mathcal{S}$. Moreover, $\Phi_{F}^{\beta}$ and $\Phi_{M}^{\beta}$ are entire analytic functions with vanishing moments of arbitrary order for $\Phi_{M}^{\beta}$ because (1.30) implies $\operatorname{supp} \varphi \subset\left\{x \in \mathbb{R}^{n}: \frac{1}{2} \leq|x|_{a} \leq \frac{3}{2}\right\}$, and thus (1.32) yields $D^{\alpha}\left(\Phi_{M}^{\beta}\right)^{\vee}(0)=0$, $\alpha \in \mathbb{N}_{0}^{n}$, which can be rewritten as

$$
\int_{\mathbb{R}^{n}} \Phi_{M}^{\beta}(\xi) \xi^{\alpha} \mathrm{d} \xi=0, \quad \alpha \in \mathbb{N}_{0}^{n} .
$$

By construction, we have

$$
\begin{array}{ll}
\Phi_{F}^{\beta}(x)=\sum_{m \in \mathbb{Z}^{n}}\left(\omega^{\beta}\right)^{\vee}(m) \widehat{\varphi_{0}}(x+m), & x \in \mathbb{R}^{n}, \\
\Phi_{M}^{\beta}(x)=\sum_{m \in \mathbb{Z}^{n}}\left(\omega^{\beta}\right)^{\vee}(m) \widehat{\varphi}(x+m), & x \in \mathbb{R}^{n} .
\end{array}
$$

For fundamentals about wavelets we refer, for instance, to [24] and [40]. 


\section{MAIN RESULTS}

Let $\Phi_{F}^{\beta}$ and $\Phi_{M}^{\beta}, \beta \in \mathbb{N}_{0}^{n}$, be given by Definition 1.11, and introduce for $j \in \mathbb{N}_{0}, m \in \mathbb{Z}^{n}$, the wavelets

$$
\Phi_{j m}^{\beta}(x)= \begin{cases}\Phi_{F}^{\beta}(x-m), & \text { if } j=0, \\ \Phi_{M}^{\beta}\left(2^{j a} x-m\right), & \text { if } j \in \mathbb{N} .\end{cases}
$$

According to the dual pairing $\left(\mathcal{S}, \mathcal{S}^{\prime}\right)$ we put, for given $f \in \mathcal{S}^{\prime}$,

$$
\lambda_{j m}^{\beta}(f)=2^{j n}\left(\Phi_{j m}^{\beta}, f\right), \quad j \in \mathbb{N}_{0}, m \in \mathbb{Z}^{n}, \beta \in \mathbb{N}_{0}^{n} .
$$

Finally, let

$$
B_{p}^{+, a}=\bigcup_{s>0} B_{p}^{s, a}, \quad 0<p \leq \infty .
$$

Recall our notation (1.17) and (1.9).

Theorem 2.1. Let $0<p \leq \infty, s>\sigma_{p}, \varrho \geq 0$, and $a$ be an anisotropy.

(i) Then $f \in \mathcal{S}^{\prime}$ is an element of $B_{p}^{s, a}$ if and only if it can be represented as

$$
f=\sum_{\beta \in \mathbb{N}_{0}^{n}} \sum_{j=0}^{\infty} \sum_{m \in \mathbb{Z}^{n}} \lambda_{j m}^{\beta} k^{\beta}\left(2^{j a} x-m\right), \quad x \in \mathbb{R}^{n},
$$

with $\left\|\lambda \mid b_{p}^{s, \varrho}\right\|<\infty$, the series being absolutely convergent in $L_{\max (1, p)}$. Moreover,

$$
\left\|f\left|B_{p}^{s, a}\|\sim \inf \| \lambda\right| b_{p}^{s, \varrho}\right\|
$$

where the infimum is taken over all admissible representations (2.3).

(ii) Let $\lambda_{j m}^{\beta}(f)$ be given by (2.2). Then $f \in B_{p}^{+, a}$ can be represented as

$$
f=\sum_{\beta \in \mathbb{N}_{0}^{n}} \sum_{j=0}^{\infty} \sum_{m \in \mathbb{Z}^{n}} \lambda_{j m}^{\beta}(f) k^{\beta}\left(2^{j a} x-m\right),
$$

the series being absolutely convergent in $L_{\max (1, p)}$, and, in addition, $f \in$ $B_{p}^{s, a}$ if and only if $\left\|\lambda(f) \mid b_{p}^{s, \varrho}\right\|<\infty$.

(iii) Let $f \in B_{p}^{s, a}$, then (2.5) is an optimal representation, i.e.,

$$
\left\|f\left|B_{p}^{s, a}\|\sim\| \lambda(f)\right| b_{p}^{s, \varrho}\right\|
$$

where the equivalence constants are independent of $f$.

Remark 2.2. The isotropic version of this result can be found in [38] which in turn is a specification and modification of [36, Theorem 2.9], where further references and approaches are discussed. Note that (i) represents the so-called sub-atomic (or quarkonial) decomposition in $B_{p}^{s, a}$; we refer also to $[13$, Theorem 3.7].

As already mentioned in the introduction, we study the "dual" situation, i.e., spaces $B_{p}^{s, a}$ with $s<0$, too. For that purpose, denote the counterpart of $B_{p}^{+, a}$ by

$$
B_{p}^{-, a}=\bigcup_{s<0} B_{p}^{s, a}, \quad 0<p \leq \infty
$$


Let $k$ and $k^{\beta}$ be given by (1.23) and (1.24), and consider the corresponding local means,

$$
k^{\beta}(t, f)(x)=\int_{\mathbb{R}^{n}} k^{\beta}(y) f\left(x+t^{a} y\right) \mathrm{d} y, \quad t>0, \quad x \in \mathbb{R}^{n},
$$

where $x+t^{a} y=\left(x_{1}+t^{a_{1}} y_{1}, \ldots, x_{n}+t^{a_{n}} y_{n}\right)$, and put

$$
k_{j m}^{\beta}(f)=k^{\beta}\left(2^{-j}, f\right)\left(2^{-j a} m\right), \quad j \in \mathbb{N}_{0}, \quad m \in \mathbb{Z}^{n} .
$$

We use the norm given by (1.26) with $\varrho=0$, denoted simply by $b_{p}^{s}=b_{p}^{s, 0}$ for convenience. Let $k(f)=\left\{k_{j m}^{\beta}(f): j \in \mathbb{N}_{0}, m \in \mathbb{Z}^{n}, \beta \in \mathbb{N}_{0}^{n}\right\}$, hence

$$
\left\|k(f) \mid b_{p}^{s}\right\|=\left(\sum_{\beta \in \mathbb{N}_{0}^{n}} \sum_{j=0}^{\infty} \sum_{m \in \mathbb{Z}^{n}} 2^{j(s-n / p) p}\left|k_{j m}^{\beta}(f)\right|^{p}\right)^{\frac{1}{p}} .
$$

Recall our notation (2.1).

Theorem 2.3. Let $1<p \leq \infty, s<0$.

(i) Then $f \in \mathcal{S}^{\prime}$ is an element of $B_{p}^{s, a}$ if and only if it can be represented as

$$
f=\sum_{\beta \in \mathbb{N}_{0}^{n}} \sum_{j=0}^{\infty} \sum_{m \in \mathbb{Z}^{n}} \lambda_{j m}^{\beta} \Phi_{j m}^{\beta}, \quad x \in \mathbb{R}^{n},
$$

with $\left\|\lambda \mid b_{p}^{s}\right\|<\infty$, the series being unconditionally convergent in $\mathcal{S}^{\prime}$. Moreover,

$$
\left\|f\left|B_{p}^{s, a}\|\sim \inf \| \lambda\right| b_{p}^{s}\right\|
$$

where the infimum is taken over all admissible representations (2.11).

(ii) Any $f \in B_{p}^{-, a}$ can be represented as

$$
f=\sum_{\beta \in \mathbb{N}_{0}^{n}} \sum_{j=0}^{\infty} \sum_{m \in \mathbb{Z}^{n}} k_{j m}^{\beta}(f) \Phi_{j m}^{\beta}
$$

the series being unconditionally convergent in $\mathcal{S}^{\prime}$, and, in addition, $f \in$ $B_{p}^{s, a}$ if and only if $\left\|k(f) \mid b_{p}^{s}\right\|<\infty$.

(iii) Let $f \in B_{p}^{s, a}$, then (2.13) is an optimal representation, i.e.,

$$
\left\|f\left|B_{p}^{s, a}\|\sim\| k(f)\right| b_{p}^{s}\right\|
$$

where the equivalence constants are independent of $f$.

Remark 2.4. Parallel to Remark 2.2 we refer to the isotropic version of the above result in [38] with further discussions (about local means) in [34] and [36]. 


\section{PROOFS}

We now collect all proofs and make use of the notation and concepts introduced before.

Proof of Proposition 1.5. We want to show that

$$
\left(\sum_{j=0}^{\infty} \sum_{m \in \mathbb{Z}^{n}} 2^{j(s-n / p) p}\left|\left(\varphi_{j}^{a} \widehat{f}\right)^{\vee}\left(2^{-j a} m\right)\right|^{p}\right)^{1 / p} \sim\left\|f \mid B_{p}^{s, a}\right\| .
$$

Taking into account definition (1.8),

$$
\left\|f \mid B_{p}^{s, a}\right\|=\left(\sum_{j=0}^{\infty} 2^{j s p}\left\|\left(\varphi_{j}^{a} \widehat{f}\right)^{\vee} \mid L_{p}\right\|^{p}\right)^{1 / p}
$$

the assertion reduces to

$$
\sum_{m \in \mathbb{Z}^{n}}\left|\left(\varphi_{j}^{a} \widehat{f}\right)^{\vee}\left(2^{-j a} m\right)\right|^{p} \sim 2^{j n}\left\|\left(\varphi_{j}^{a} \widehat{f}\right)^{\vee} \mid L_{p}\right\|^{p}
$$

with equivalence constants independent of $j \in \mathbb{N}_{0}$ and $f \in \mathcal{S}^{\prime}$. Here we use an isotropic result given in [33, Section 1.3.3]: adapted to our above notation, it states that for $0<p \leq \infty$ there exist some numbers $\nu_{0}>0$ and $c_{2}>c_{1}>0$ such that for all $\nu \geq \nu_{0}$, and all $\varphi \in \mathcal{S}$ with $\operatorname{supp} \mathcal{F} \varphi \subset \Omega$ there holds

$$
c_{1} \sum_{m \in \mathbb{Z}^{n}}\left|\varphi\left(2^{-\nu} m\right)\right|^{p} \leq 2^{\nu n}\left\|\left.\varphi\left|L_{p} \|^{p} \leq c_{2} \sum_{m \in \mathbb{Z}^{n}}\right| \varphi\left(2^{-\nu} m\right)\right|^{p},\right.
$$

where $\Omega \subset \mathbb{R}^{n}$ is compact. (This should be properly modified when $p=\infty$.) In addition, it is known, cf. [33, Remark 1.3.3] or [32, 1.3.5], that if for some suitably chosen $y^{0} \in \mathbb{R}^{n}$ and $b>0$,

$$
\Omega \subset\left\{y \in \mathbb{R}^{n}:\left|y_{j}-y_{j}^{0}\right| \leq b, j=1, \ldots, n\right\},
$$

then $\nu_{0}$ can be taken such that $2^{\nu_{0}} \sim b$. Thus, with $\varphi=\left(\varphi_{j} \widehat{f}\right)^{\vee}$ this implies $b \sim 2^{j}$ in the isotropic case, i.e., (3.4) with $\varphi=\left(\varphi_{j} \widehat{f}\right)^{\vee}$ and $\nu=j$ yields the desired (isotropic) result. In order to prove (3.3) we slightly modify the above argument: let $\psi \in \mathcal{S}$ with

$$
\operatorname{supp} \mathcal{F} \psi \subset \Omega^{a} \subset\left\{y \in \mathbb{R}^{n}:\left|y_{j}-y_{j}^{1}\right| \leq b^{a_{j}}, j=1, \ldots, n\right\},
$$

for suitably chosen $y^{1} \in \mathbb{R}^{n}$ and $b>0$, then we define

$$
\varphi(t x):=\psi\left(t^{a} x\right), \quad t>0, \quad x \in \mathbb{R}^{n} .
$$

Obviously, $\varphi \in \mathcal{S}, \mathcal{F} \varphi(t \xi)=\mathcal{F} \psi\left(t^{a} \xi\right), \quad t>0, \quad \xi \in \mathbb{R}^{n}$, and consequently

$$
\operatorname{supp} \mathcal{F} \varphi \subset\left\{y \in \mathbb{R}^{n}:\left|y_{j}-y_{j}^{0}\right| \leq b, j=1, \ldots, n\right\}
$$

(with $y^{0}=b^{1-a} y^{1}$ ). Hence the application of (3.4) and (3.6) leads to

$$
c_{1} \sum_{m \in \mathbb{Z}^{n}}\left|\psi\left(2^{-\nu a} m\right)\right|^{p} \leq 2^{\nu n}\left\|\left.\varphi\left|L_{p} \|^{p} \leq c_{2} \sum_{m \in \mathbb{Z}^{n}}\right| \psi\left(2^{-\nu a} m\right)\right|^{p} .\right.
$$


On the other hand, with $a_{1}+\cdots+a_{n}=n$,

$$
\begin{aligned}
\left\|\varphi \mid L_{p}\right\|^{p} & =\int_{\mathbb{R}^{n}}|\varphi(x)|^{p} \mathrm{~d} x=t^{n} \int_{\mathbb{R}^{n}}|\varphi(t y)|^{p} \mathrm{~d} y \\
& =t^{n} \int_{\mathbb{R}^{n}}\left|\psi\left(t^{a} y\right)\right|^{p} \mathrm{~d} y=\int_{\mathbb{R}^{n}}|\psi(z)|^{p} \mathrm{~d} z=\left\|\psi \mid L_{p}\right\|^{p} .
\end{aligned}
$$

Finally, (3.7) and (3.8) with $\psi=\left(\varphi_{j}^{a} \widehat{f}\right)^{\vee}, b \sim 2^{j}$ and $\nu=j$ finish the proof.

Proof of Theorem 2.1.

Step 1. We assume that $f$ is given by (2.3) with $\left\|\lambda \mid b_{p}^{s, \varrho}\right\|<\infty$ for some $\varrho \geq 0$. We want to show that $f \in B_{p}^{s, a}$ and that there exists a constant $c>0$ such that

$$
\left\|f\left|B_{p}^{s, a}\|\leq c\| \lambda\right| b_{p}^{s, \varrho}\right\|
$$

We rewrite $(2.3)$ as

$$
f=\sum_{\beta} f^{\beta} \quad \text { with } \quad f^{\beta}=\sum_{j, m} \lambda_{j, m}^{\beta} k^{\beta}\left(2^{j a} x-m\right) .
$$

By definition (1.22), the support of $k$ is contained in an open ball centered at the origin and of radius $2^{J-\varepsilon}$ for some $\varepsilon>0$. Using the atomic decomposition for the spaces $B_{p}^{s, a}$ with $0<p \leq \infty$ and $s>\sigma_{p}$ described in Theorem 1.9, we find by Definition 1.6 that

$$
\left\{2^{\varepsilon a \beta} 2^{-j(s-n / p)} k^{\beta}\left(2^{j a} x-m\right): \quad j \in \mathbb{N}_{0}, \quad m \in \mathbb{Z}^{n}\right\}, \quad \beta \in \mathbb{N}_{0}^{n},
$$

are admissible systems of anisotropic atoms, and hence $f^{\beta} \in B_{p}^{s, a}$ for all $\beta \in \mathbb{N}_{0}^{n}$, with

$$
\left\|f^{\beta} \mid B_{p}^{s, a}\right\| \leq c 2^{-\varepsilon a \beta}\left(\sum_{j=0}^{\infty} \sum_{m \in \mathbb{Z}^{n}} 2^{j(s-n / p) p}\left|\lambda_{j m}^{\beta}\right|^{p}\right)^{1 / p},
$$

and $c$ independent of $\beta \in \mathbb{N}_{0}^{n}$. Summation over $\beta$ proves $f \in B_{p}^{s, a}$ and (3.9). The absolute convergence follows in the same way as discussed in [38] and [36, $1.4,2.7]$.

Step 2. Let $f \in B_{p}^{s, a}$ with $0<p \leq \infty$ and $s>\sigma_{p}$; we shall show that we can decompose it as (2.5). Note that this covers then (i) as well.

Let $R_{j}^{a}, j \in \mathbb{N}_{0}$, be a rectangle in $\mathbb{R}^{n}$ centered at the origin with side-length $2 \pi 2^{j a}$ where $2^{j a}=\left(2^{j a_{1}}, \ldots, 2^{j a_{n}}\right)$. Let $\varphi_{j}^{a}$ be given by $(1.7)$, that is, with $\operatorname{supp} \varphi_{j}^{a} \subset R_{j}^{a}$. Now we can write that

$$
\widehat{f}(x)=\sum_{j=0}^{\infty} \varphi_{j}^{a}(x) \widehat{f}(x), \quad x \in \mathbb{R}^{n},
$$

and expand $\varphi_{j}^{a} \widehat{f}$ in $R_{j}^{a}$ into a Fourier series,

$$
\left(\varphi_{j}^{a} \widehat{f}\right)(\xi)=\sum_{m \in \mathbb{Z}^{n}} b_{j m} \exp \left(-i 2^{-j a} m \xi\right), \quad \xi \in R_{j}^{a} .
$$


We calculate $b_{j m}, j \in \mathbb{N}_{0}, m \in \mathbb{Z}^{n}$, by

$$
\begin{aligned}
\left(\varphi_{j}^{a} \widehat{f}\right)^{\vee}\left(2^{-j a} m\right) & =(2 \pi)^{-\frac{n}{2}} \int_{R_{j}^{a}}\left(\varphi_{j}^{a} \widehat{f}\right)(y) \exp \left(i 2^{-j a} m y\right) \mathrm{d} y \\
& =(2 \pi)^{-\frac{n}{2}} \sum_{k \in \mathbb{Z}^{n}} b_{j k} \int_{R_{j}^{a}} \exp \left(i 2^{-j a}(m-k) y\right) \mathrm{d} y \\
& =(2 \pi)^{-\frac{n}{2}} \sum_{k \in \mathbb{Z}^{n}} b_{j k} \int_{R_{j}^{a}} \exp \left(i \sum_{l=1}^{n} 2^{-j a_{l}}\left(m_{l}-k_{l}\right) y_{l}\right) \mathrm{d} y .
\end{aligned}
$$

Substitute in the last formula $\xi_{l}=2^{-j a_{l}} y_{l}$, then $\mathrm{d} \xi=2^{-j\left(a_{1}+\cdots+a_{n}\right)} \mathrm{d} y=$ $2^{-j n} \mathrm{~d} y$, and we arrive at

$$
\left(\varphi_{j}^{a} \widehat{f}\right)^{\vee}\left(2^{-j a} m\right)=2^{j n}(2 \pi)^{-\frac{n}{2}} \sum_{k \in \mathbb{Z}^{n}} b_{j k} \int_{Q_{\pi}} e^{i(m-k) \xi} \mathrm{d} \xi
$$

where $Q_{\pi}$ is the cube of side-length $2 \pi$ in each direction. For the last term in (3.14) we thus have

$$
\int_{Q_{\pi}} e^{i(m-k) \xi} \mathrm{d} \xi= \begin{cases}(2 \pi)^{n}, & m=k \\ 0, & m \neq k\end{cases}
$$

which by (3.14) finally leads to

$$
\begin{aligned}
b_{j m} & =(2 \pi)^{-\frac{n}{2}} 2^{-j n}\left(\varphi_{j}^{a} \widehat{f}\right)^{\vee}\left(2^{-j a} m\right) \\
& =(2 \pi)^{-n} 2^{-j n} \int_{R_{j}^{a}}\left(\varphi_{j}^{a} \widehat{f}\right)(\xi) \exp \left(i 2^{-j a} m \xi\right) \mathrm{d} \xi .
\end{aligned}
$$

By Proposition 1.5 and (3.15) we thus have for $0<p \leq \infty$,

$$
\left\|f \mid B_{p}^{s, a}\right\| \sim\left(\sum_{j=0}^{\infty} 2^{j(s-n / p) p} 2^{j n p} \sum_{m \in \mathbb{Z}^{n}}\left|b_{j m}\right|^{p}\right)^{1 / p}
$$

(with the usual modification if $p=\infty$ ). Let $\omega$ be given by $(1.27)$ and $\omega_{j}(x)=$ $\omega\left(2^{-j a} x\right)$; then $\omega_{j}$ has a compact support in $R_{j}^{a}$ and it follows by (3.13) that

$$
\begin{aligned}
\left(\varphi_{j}^{a} \widehat{f}\right)^{\vee}(x) & =\sum_{m \in \mathbb{Z}^{n}} b_{j m} \omega_{j}^{\vee}\left(x-2^{-j a} m\right) \\
& =2^{j n} \sum_{m \in \mathbb{Z}^{n}} b_{j m} \omega^{\vee}\left(2^{j a} x-m\right), \quad x \in \mathbb{R}^{n} .
\end{aligned}
$$

Let $k$ be given by $(1.22),(1.23)$. We expand the analytic function $\omega^{\vee}\left(2^{j a} x-m\right)$ at $2^{-j a} l, l \in \mathbb{Z}^{n}$, and obtain

$$
k\left(2^{j a} x-l\right) \omega^{\vee}\left(2^{j a} x-m\right)=\sum_{\beta \in \mathbb{N}_{0}^{n}} \frac{2^{j a \beta}}{\beta !}\left(D^{\beta} \omega^{\vee}\right)(l-m)\left(x-2^{-j a} l\right)^{\beta} k\left(2^{j a} x-l\right)
$$




$$
\begin{aligned}
& =\sum_{\beta \in \mathbb{N}_{0}^{n}} \frac{2^{j a \beta}}{\beta !}\left(D^{\beta} \omega^{\vee}\right)(l-m) 2^{-j a \beta}\left(2^{j a} x-l\right)^{\beta} k\left(2^{j a} x-l\right) \\
& =\sum_{\beta \in \mathbb{N}_{0}^{n}} \frac{2^{J a \beta}}{\beta !}\left(D^{\beta} \omega^{\vee}\right)(l-m) k^{\beta}\left(2^{j a} x-l\right),
\end{aligned}
$$

where we applied (1.24) to the last line. By (1.23), (3.17) and (3.18) we obtain

$$
\begin{aligned}
\left(\varphi_{j}^{a} \widehat{f}\right)^{\vee}(x) & =\sum_{m \in \mathbb{Z}^{n}} 2^{j n} b_{j m} \sum_{l \in \mathbb{Z}^{n}} k\left(2^{j a} x-l\right) \omega^{\vee}\left(2^{j a} x-m\right) \\
& =\sum_{\beta \in \mathbb{N}_{0}^{n}} \sum_{l \in \mathbb{Z}^{n}} k^{\beta}\left(2^{j a} x-l\right) \sum_{m \in \mathbb{Z}^{n}} 2^{j n} b_{j m} \frac{2^{J a \beta}}{\beta !}\left(D^{\beta} \omega^{\vee}\right)(l-m) .
\end{aligned}
$$

Hence, as $\left(\varphi_{j}^{a}\right)_{j \in \mathbb{N}_{0}}$ is a resolution of unity,

$$
f=\sum_{j=0}^{\infty} \sum_{\beta \in \mathbb{N}_{0}^{n}} \sum_{l \in \mathbb{Z}^{n}} k^{\beta}\left(2^{j a} x-l\right) \lambda_{j l}^{\beta}
$$

with

$$
\lambda_{j l}^{\beta}=\sum_{m \in \mathbb{Z}^{n}} 2^{j n} b_{j m} \frac{2^{J a \beta}}{\beta !}\left(D^{\beta} \omega^{\vee}\right)(l-m) .
$$

We first check that $\lambda_{j l}^{\beta}$ are optimal coefficients, and verify their representation (2.2) afterwards. We thus claim that for $\varrho \geq 0$ we can find a constant $c$ such that with $\lambda=\left\{\lambda_{j l}^{\beta}: \beta \in \mathbb{N}_{0}^{n}, j \in \mathbb{N}_{0}, l \in \mathbb{Z}^{n}\right\}$ given by (3.20),

$$
\left\|\lambda\left|b_{p}^{s, \varrho}\|\leq c\| f\right| B_{p}^{s, a}\right\| \quad \text { for all } \quad f \in B_{p}^{s, a} .
$$

We use an isotropic result [37, 3.1.1], which states that for any $\varepsilon>0$ there are constants $c>0$ and $c_{\varepsilon}>0$ such that

$$
\left|D^{\beta} \omega^{\vee}(x)\right| \leq c_{\varepsilon} 2^{c|\beta|}\left(1+|x|^{2}\right)^{-\varepsilon} \quad \text { for } \quad x \in \mathbb{R}^{n}, \quad \beta \in \mathbb{N}_{0}^{n},
$$

where $c$ is independent of $x, \varepsilon$, and $\beta$, and $c_{\varepsilon}$ independent of $x, \beta$. Furthermore, note that there are constants $c_{2}>c_{1}>0$ such that for all $\xi \in \mathbb{R}^{n}$,

$$
c_{1}(1+|\xi|)^{1 / a_{\max }} \leq 1+|\xi|_{a} \leq c_{2}(1+|\xi|)^{1 / a_{\min }},
$$

cf. [23]. On the other hand, we have $a_{\min }|\beta| \leq a \beta \leq a_{\max }|\beta|$, thus (3.22) implies

$$
\left|D^{\beta} \omega^{\vee}(x)\right| \leq c_{\varepsilon}^{\prime} 2^{c^{\prime} a \beta}\left(1+|x|_{a}\right)^{-\varepsilon} \quad \text { for } \quad x \in \mathbb{R}^{n}, \quad \beta \in \mathbb{N}_{0}^{n} .
$$

Let $p \geq 1$. We interpret $\lambda_{j l}^{\beta}$ as a convolution in $\ell_{p}$ : let $l \in \mathbb{Z}^{n}$ and

$$
a_{l}=\sum_{m \in \mathbb{Z}^{n}} c_{m} d_{l-m}
$$

then $\left\|a_{l}\left|\ell_{p}\|\leq\| d_{k}\right| \ell_{1}\right\|\left\|c_{m} \mid \ell_{p}\right\|$. Put $d_{k}=\frac{2^{J a \beta}}{\beta !}\left(D^{\beta} \omega^{\vee}\right)(k)$, then (3.23) leads to

$$
\left\|d_{k} \mid \ell_{1}\right\| \leq C_{\varepsilon} \frac{2^{\left(J+c^{\prime}\right) a \beta}}{\beta !} \leq c(\varrho) 2^{-(\varrho+1) a \beta}
$$


if $\varepsilon>0$ is chosen appropriately. The last inequality results from an estimate of $\beta !=\beta_{1} ! \cdots \beta_{n}$ ! by Stirling's formula, $n !=\Gamma(n+1) \sim \sqrt{n}\left(\frac{n}{e}\right)^{n}, n \in \mathbb{N}$. Consequently, (3.20) with $a_{l}=\lambda_{j l}^{\beta}$ and $c_{m}=2^{j n} b_{j m}$ implies, that for $\varrho \geq 0$ there is a constant $c(\varrho)$ such that

$$
\left(\sum_{l \in \mathbb{Z}^{n}}\left|\lambda_{j l}^{\beta}\right|^{p}\right)^{1 / p} \leq c(\varrho) 2^{-(\varrho+1) a \beta}\left(\sum_{l \in \mathbb{Z}^{n}}\left|2^{j n} b_{j l}\right|^{p}\right)^{1 / p} .
$$

If $p<1$, then one uses the $p$-triangle inequality. Now (3.21) follows from (3.24) and (3.16).

Step 3. We need to prove that $\lambda_{j l}^{\beta}$ can be represented as (2.2). By (3.15) and the properties of the Fourier transform we have

$$
2^{j n} b_{j m}=(2 \pi)^{-n} \int_{\mathbb{R}^{n}}\left(\varphi_{j}^{a}\right)^{\vee}\left(2^{-j a} m-y\right) f(y) \mathrm{d} y, \quad j \in \mathbb{N}_{0},
$$

where $\varphi$ is given by Definition 1.11. Now $\varphi_{j}^{a}(x)=\varphi\left(2^{-j a} x\right)$ leads to

$$
2^{j n} b_{j m}=(2 \pi)^{-n} 2^{j n} \int_{\mathbb{R}^{n}} \varphi^{\vee}\left(m-2^{-j a} y\right) f(y) \mathrm{d} y, \quad j \in \mathbb{N} .
$$

Recall that $\left(D^{\beta} \omega^{\vee}\right)(\xi)=i^{|\beta|}\left(x^{\beta} \omega(x)\right)^{\vee}(\xi)$. Thus (1.28) implies for $j \in \mathbb{N}$,

$$
\begin{aligned}
\lambda_{j l}^{\beta} & =\sum_{m \in \mathbb{Z}^{n}} 2^{j n} b_{j m} \frac{2^{J a \beta}}{\beta !}\left(D^{\beta} \omega^{\vee}\right)(l-m) \\
& =\sum_{m \in \mathbb{Z}^{n}} 2^{j n} b_{j m} \frac{2^{J a \beta}}{\beta !} i^{|\beta|}\left(x^{\beta} \omega(x)\right)^{\vee}(l-m) \\
& =\sum_{m \in \mathbb{Z}^{n}} 2^{j n} b_{j m} \frac{2^{J a \beta}}{\beta !} i^{|\beta|} \frac{(2 \pi)^{n} \cdot \beta !}{i^{|\beta|} 2^{J a \beta}}\left(\omega^{\beta}\right)^{\vee}(l-m) \\
& =2^{j n} \int_{\mathbb{R}^{n}} f(y) \sum_{m \in \mathbb{Z}^{n}}\left(\omega^{\beta}\right)^{\vee}(l-m) \varphi^{\vee}\left(m-2^{j a} y\right) \mathrm{d} y .
\end{aligned}
$$

Replacing $l-m$ by $m$ and using $\varphi^{\vee}(z)=\widehat{\varphi}(-z)$ we get

$$
\lambda_{j l}^{\beta}=2^{j n} \int_{\mathbb{R}^{n}} f(y) \sum_{m \in \mathbb{Z}^{n}}\left(\omega^{\beta}\right)^{\vee}(m) \widehat{\varphi}\left(2^{j a} y-l+m\right) \mathrm{d} y=2^{j n}\left(\Phi_{j l}^{\beta}, f\right), \quad j \in \mathbb{N},
$$

where we used (1.35) and (2.1). The argument for $j=0$ works analogously, so the proof is finished.

We begin the proof of Theorem 2.3 with some preparation. Let $l \in \mathbb{Z}^{n}$, $K, L \in \mathbb{R}$, and $a_{l} \in C^{K}$ be anisotropic atoms given by Definition 1.6 with $\operatorname{supp} a_{l} \subset\left\{y \in \mathbb{R}^{n}:|y|_{a} \leq c\right\}$ for some appropriate $c>0$. We know by (1.15) that

$$
\int_{\mathbb{R}^{n}} x^{\beta} a_{l}(x) \mathrm{d} x=0 \quad \text { if } \quad a \beta \leq L .
$$


Let $\mu=\left(\mu_{l}\right)_{l \in \mathbb{Z}^{n}}$ denote the decay factors,

$$
\left|D^{\gamma} a_{l}(x)\right| \leq \mu_{l}, \quad a \gamma \leq K, \quad x \in \mathbb{R}^{n} .
$$

We define now (special) anisotropic molecules

$$
b(x)=\sum_{l \in \mathbb{Z}^{n}} a_{l}(x-l), \quad x \in \mathbb{R}^{n},
$$

and

$$
b_{j, m}(x)=2^{-j\left(s-\frac{n}{p}\right)} b\left(2^{j a} x-m\right), \quad j \in \mathbb{N}_{0}, m \in \mathbb{Z}^{n}, x \in \mathbb{R}^{n} .
$$

Remark 3.1. The normalized (isotropic) molecules share the decay properties and moment conditions with the normalized (isotropic) atoms, but lack the assumption concerning a compact support, see (the isotropic counterparts of) (1.11), (1.13). This assumption is replaced by sufficiently strong decay assumption. In the isotropic case the counterpart of Theorem 1.9 remains valid if one uses molecular decompositions instead of atomic ones, cf. [16, Section 5]. There are also anisotropic versions of that result in the literature, see [7]. However, because of the special property of building blocks we shall use, we do not need this assertion in its full generality, but only a special case which is simpler to prove. Hence we consider this case below separately and give its direct proof.

Proposition 3.2. Let $s \in \mathbb{R}, 0<p \leq \infty, 0<q \leq \infty$, and $\mu=\left(\mu_{l}\right)_{l \in \mathbb{Z}^{n}} \in$ $\ell_{\min (1, p)}$. Let $f \in \mathcal{S}^{\prime}$ be given by

$$
f=\sum_{j=0}^{\infty} \sum_{m \in \mathbb{Z}^{n}} \lambda_{j, m} b_{j, m},
$$

where $\lambda=\left\{\lambda_{j, m} \in \mathbb{C}: j \in \mathbb{N}_{0}, m \in \mathbb{Z}^{n}\right\}, \lambda \in b_{p q}$, and $b_{j, m}, j \in \mathbb{N}_{0}, m \in \mathbb{Z}^{n}$, are given by (3.32). Then

$$
\left\|f\left|B_{p q}^{s, a}\|\leq c\| \lambda\right| b_{p q}\right\| .
$$

Proof. We owe the idea of this proof to some discussions with Prof. H. Triebel.

By definition and (3.31), (3.32),

$$
\begin{aligned}
f & =\sum_{j=0}^{\infty} \sum_{m \in \mathbb{Z}^{n}} \lambda_{j, m} b_{j, m} \\
& =\sum_{j=0}^{\infty} 2^{-j\left(s-\frac{n}{p}\right)} \sum_{m \in \mathbb{Z}^{n}} \lambda_{j, m} b\left(2^{j a} x-m\right) \\
& =\sum_{j=0}^{\infty} 2^{-j\left(s-\frac{n}{p}\right)} \sum_{m \in \mathbb{Z}^{n}} \lambda_{j, m} \sum_{l \in \mathbb{Z}^{n}} a_{l}\left(2^{j a} x-m-l\right) \\
& =\sum_{j=0}^{\infty} 2^{-j\left(s-\frac{n}{p}\right)} \sum_{k \in \mathbb{Z}^{n}} \sum_{l \in \mathbb{Z}^{n}} \lambda_{j, k-l} a_{l}\left(2^{j a} x-k\right) \\
& =\sum_{j=0}^{\infty} 2^{-j\left(s-\frac{n}{p}\right)} \sum_{k \in \mathbb{Z}^{n}} \gamma_{j, k} d_{j, k}(x),
\end{aligned}
$$


where we have put

$$
\gamma_{j, k}=\sum_{l \in \mathbb{Z}^{n}} \mu_{l}\left|\lambda_{j, k-l}\right|>0, \quad j \in \mathbb{N}_{0}, k \in \mathbb{Z}^{n},
$$

and

$$
d_{j, k}(x)=\gamma_{j, k}^{-1} \sum_{l \in \mathbb{Z}^{n}} \lambda_{j, k-l} a_{l}\left(2^{j a} x-k\right), \quad j \in \mathbb{N}_{0}, k \in \mathbb{Z}^{n}, x \in \mathbb{R}^{n} .
$$

We claim that $2^{-j\left(s-\frac{n}{p}\right)} d_{j, k}$ are anisotropic atoms according to Definition 1.6. Assume first $j=0$, then

$$
\left|d_{0, k}(x)\right| \leq \gamma_{0, k}^{-1} \sum_{l \in \mathbb{Z}^{n}}\left|\lambda_{0, k-l}\right| \cdot \mu_{l}=1
$$

and likewise for all derivatives $D^{\gamma} d_{0, k}, \gamma \in \mathbb{N}_{0}^{n}, a \gamma \leq K$, according to (3.30). In the case of $j \in \mathbb{N}$ and $\gamma \in \mathbb{N}_{0}^{n}$ we conclude similarly that

$$
\begin{aligned}
\left|D^{\gamma}\left(2^{-j\left(s-\frac{n}{p}\right)} d_{j, k}(x)\right)\right| & \leq 2^{-j\left(s-\frac{n}{p}\right)} \gamma_{j, k}^{-1} \sum_{l \in \mathbb{Z}^{n}}\left|\lambda_{j, k-l}\right|\left|\left(D^{\gamma} a_{l}\right)\left(2^{j a} x-k\right)\right| 2^{j a \gamma} \\
& \leq 2^{-j\left(s-\frac{n}{p}\right)+j a \gamma} \gamma_{j, k}^{-1} \sum_{l \in \mathbb{Z}^{n}}\left|\lambda_{j, k-l}\right| \mu_{l}=2^{-j\left(s-\frac{n}{p}\right)+j a \gamma}
\end{aligned}
$$

where $a \gamma \leq K$. The corresponding moment conditions (1.15) are satisfied by (3.29), and condition (1.13) is guaranteed by construction (3.36). Hence $2^{-j\left(s-\frac{n}{p}\right)} d_{j, k}$ are anisotropic atoms and Theorem 1.9 gives the anisotropic atomic decomposition

$$
f=\sum_{j=0}^{\infty} \sum_{k \in \mathbb{Z}^{n}} \gamma_{j, k} 2^{-j\left(s-\frac{n}{p}\right)} d_{j, k} \quad \text { with } \quad\left\|f\left|B_{p q}^{s, a}\|\leq c\| \gamma\right| b_{p q}\right\| .
$$

It remains to estimate $\left\|\gamma \mid b_{p q}\right\|$ by $\left\|\lambda \mid b_{p q}\right\|$, where the assumption on the decay factors, i.e., $\mu \in \ell_{\min (p, 1)}$ is now involved. We first assume that $p \geq 1$, then interpret $\gamma_{j, k}$ as a convolution in $\ell_{p}$ and obtain

$$
\left(\sum_{k \in \mathbb{Z}^{n}}\left|\gamma_{j, k}\right|^{p}\right)^{\frac{1}{p}} \leq\left(\sum_{l \in \mathbb{Z}^{n}} \mu_{l}\right)\left(\sum_{m \in \mathbb{Z}^{n}}\left|\lambda_{j, m}\right|^{p}\right)^{\frac{1}{p}}=\left\|\mu \mid \ell_{1}\right\|\left(\sum_{m \in \mathbb{Z}^{n}}\left|\lambda_{j, m}\right|^{p}\right)^{\frac{1}{p}} .
$$

If $0<p<1$, we have by the $p$-triangle inequality

$$
\sum_{k \in \mathbb{Z}^{n}}\left|\gamma_{j, k}\right|^{p} \leq \sum_{k, l \in \mathbb{Z}^{n}} \mu_{l}^{p}\left|\lambda_{j, k-l}\right|^{p} \leq\left\|\left.\mu\left|\ell_{p} \|^{p} \sum_{m \in \mathbb{Z}^{n}}\right| \lambda_{j, m}\right|^{p} .\right.
$$

This results in

$$
\left\|\gamma\left|b_{p q}\|\leq c\| \mu\right| \ell_{\min (1, p)}\right\|\left\|\lambda \mid b_{p q}\right\|
$$

as desired, i.e., $\left\|f\left|B_{p q}^{s, a}\|\leq c\| \gamma\right| b_{p q}\right\| \leq c^{\prime}\left\|\mu\left|\ell_{\min (1, p)}\|\| \lambda\right| b_{p q}\right\|$.

\section{Proof of Theorem 2.3.}

Step 1. We assume that $f$ is given by (2.11) with $\left\|\lambda \mid b_{p}^{s}\right\|<\infty$. We want to show that $f \in B_{p}^{s, a}$ and that there exists a constant $c>0$ such that

$$
\left\|f\left|B_{p}^{s, a}\|\leq c\| \lambda\right| b_{p}^{s}\right\|
$$


Recall our particular construction (1.34), (1.35), (2.1) with $\varphi \in \mathcal{S}$ given by Definition 1.11. Then for each $\beta \in \mathbb{N}_{0}^{n}$,

$$
\left\{2^{\varepsilon a \beta} 2^{-j\left(s-\frac{n}{p}\right)} \tilde{\Phi}_{j m}^{\beta}: \quad j \in \mathbb{N}_{0}, \quad m \in \mathbb{Z}^{n}\right\}
$$

where $\tilde{\Phi}_{j m}^{\beta}=\sum_{l \in \mathbb{Z}^{n}} k(x-l) \Phi_{j m}^{\beta}$, are admissible anisotropic special molecules in $B_{p}^{s, a}$ in the sense of (3.29)-(3.32), satisfying, in addition, the decay assumption $\mu \in \ell_{\min (p, 1)}$. Thus we can apply Proposition 3.2 with $q=p$ and then (3.37) follows in the same way as in Step 1 in the proof of Theorem 2.1. This covers the unconditional convergence, too.

Step 2. Let $k(f)=\left\{k_{j m}^{\beta}(f): \beta \in \mathbb{N}_{0}^{n}, j \in \mathbb{N}_{0}, m \in \mathbb{Z}^{n}\right\}$ be given by (2.8), (2.9), i.e.,

$$
\begin{aligned}
k_{j m}^{\beta}(f) & =k^{\beta}\left(2^{-j}, f\right)\left(2^{-j a} m\right)=\int_{\mathbb{R}^{n}} k^{\beta}(y) f\left(2^{-j a} m+2^{-j a} y\right) \mathrm{d} y \\
& =2^{j n} \int_{\mathbb{R}^{n}} k^{\beta}\left(2^{j a} y-m\right) f(y) \mathrm{d} y=2^{j n}\left(k^{\beta}\left(2^{j a} \cdot-m\right), f\right) .
\end{aligned}
$$

We are to show that there is some $c>0$ such that for all $f \in B_{p}^{s, a}$,

$$
\left\|k(f)\left|b_{p}^{s}\|\leq c\| f\right| B_{p}^{s, a}\right\|
$$

Using (2.10) with $\varrho=0$ (recall our convention $\left.b_{p}^{s}=b_{p}^{s, 0}\right)$, (3.39) can be rewritten as

$$
\begin{aligned}
\left\|k(f) \mid b_{p}^{s}\right\| & =\left\|\sum_{\beta, j, m} 2^{j\left(s-\frac{n}{p}\right)} k_{j m}^{\beta} \mid \ell_{p}\right\| \\
& =\left\|\sum_{\beta, j, m} 2^{j\left(s-\frac{n}{p}\right)+j n}\left(k^{\beta}\left(2^{j a} \cdot-m\right), f\right) \mid \ell_{p}\right\| .
\end{aligned}
$$

By duality, $\ell_{p}=\left(\ell_{p^{\prime}}\right)^{\prime}$ with $\frac{1}{p}+\frac{1}{p^{\prime}}=1$ for $1<p \leq \infty$. Taking additionally into account that $2^{j\left(s-\frac{n}{p}\right)+j n}=2^{j\left(s+\frac{n}{p^{\prime}}\right)}$, we are led to

$$
\begin{aligned}
\left\|k(f) \mid b_{p}^{s}\right\| & =\left\|\sum_{\beta, j, m} 2^{j\left(s+\frac{n}{p^{\prime}}\right)}\left(k^{\beta}\left(2^{j a} \cdot-m\right), f\right) \mid \ell_{p}\right\| \\
& =\sup _{\lambda \mid b_{p^{\prime}}^{-s} \leq 1} \sum_{\beta, j, m} \lambda_{j m}^{\beta}\left(k^{\beta}\left(2^{j a} \cdot-m\right), f\right),
\end{aligned}
$$

where the supremum in (3.42) is taken over all sequences $\lambda=\left\{\lambda_{j m}^{\beta}: \beta \in\right.$ $\left.\mathbb{N}_{0}^{n}, j \in \mathbb{N}_{0}, m \in \mathbb{Z}^{n}\right\}$ such that the right-hand side of (3.42) is non-negative, and $\left\|\lambda \mid b_{p^{\prime}}^{-s}\right\| \leq 1$. Consequently,

$$
\left\|k(f)\left|b_{p}^{s} \| \leq \sup _{\substack{\lambda \mid b_{p^{\prime}}^{-s} \leq 1 \\ \text { s. }}}\right|(g, f) \mid, \quad \text { where } g(x)=\sum_{\beta, j, m} \lambda_{j m}^{\beta} k^{\beta}\left(2^{j a} x-m\right) .\right.
$$


Note that our assumptions $s<0$ and $1<p \leq \infty$ imply $-s>0=\sigma_{p^{\prime}}$ such that Theorem 2.1 can be applied to $g \in B_{p^{\prime}}^{-s, a}$. Thus we arrive at

$$
\left\|k(f) \mid b_{p}^{s}\right\| \leq \sup \left\{|(g, f)|: g \in B_{p^{\prime}}^{-s, a},\left\|g \mid B_{p^{\prime}}^{-s, a}\right\| \leq c\right\},
$$

where $c>0$ is independent of $g \in B_{p^{\prime}}^{-s, a}$. Now we use the duality

$$
\left(B_{p^{\prime}}^{-\sigma, a}\left(\mathbb{R}^{n}\right)\right)^{\prime}=B_{p}^{\sigma, a}\left(\mathbb{R}^{n}\right), \quad 1 \leq p^{\prime}<\infty, \quad \sigma \in \mathbb{R}
$$

see [39], and obtain

$$
\left\|k(f)\left|b_{p}^{s}\left\|\leq c^{\prime}\right\| f\right| B_{p}^{s, a}\right\|
$$

Step 3. Let $1<p \leq \infty, s<0$ and $f \in B_{p}^{s, a}$. It is sufficient to verify (2.13) in order to complete the proof. Let $\psi \in \mathcal{S}$ be arbitrary, then Theorem 2.1, in particular (2.5) with (2.2), yields

$$
\psi=\sum_{\beta, j, m} 2^{j n}\left(\Phi_{j m}^{\beta}, \psi\right) k^{\beta}\left(2^{j a} x-m\right)
$$

with unconditional convergence in any space $B_{p^{\prime}}^{\sigma, a}$ with $\sigma>0$. Hence, by (3.39),

$$
(f, \psi)=\left(\sum_{\beta, j, m} 2^{j n}\left(f, k^{\beta}\left(2^{j a} \cdot-m\right)\right) \Phi_{j m}^{\beta}, \psi\right)=\left(\sum_{\beta, j, m} k_{j m}^{\beta}(f) \Phi_{j m}^{\beta}, \psi\right),
$$

that is

$$
f=\sum_{\beta, j, m} k_{j m}^{\beta}(f) \Phi_{j m}^{\beta}
$$

in $\mathcal{S}^{\prime}$. By (i) and our preceding remarks it follows that (2.13) converges unconditionally in $\mathcal{S}^{\prime}$.

\section{ACKNOWLEDGEMENT}

We are grateful to Prof. H. Triebel for many inspiring discussions and valuable remarks concerning the subject.

The first author was supported by the Heisenberg grant HA 2794/1-1. This work was also supported by the Junior Research Team "Fractal Analysis" of the Friedrich-Schiller-University Jena financed by the Free State of Thuringia.

\section{REFERENCES}

1. D. R. Adams and L. I. Hedberg, Function spaces and potential theory. Grundlehren der Mathematischen Wissenschaften [Fundamental Principles of Mathematical Sciences], 314. Springer-Verlag, Berlin, 1996.

2. M. Z. BerkolaĬKo and I. YA. Novikov, Unconditional bases in spaces of functions of anisotropic smoothness. (Russian) Trudy Mat. Inst. Steklov. 204(1993), Issled. po Teor. Differ. Funktsii Mnogikh Peremen. i ee Prilozh. 16, 35-51; English transl.: Proc. Steklov Inst. Math. 1994, no. 3 (204), 27-41.

3. M. Z. BerkolăKo and I. YA. Novikov, Bases of wavelets, and linear operators in anisotropic Lizorkin-Triebel spaces. (Russian) Trudy Mat. Inst. Steklov. 210(1995), Teor. Funktsii $i$ Differ. Uravn., 5-30.

4. O. V. Besov, V. P. IL'In, and S. M. NiKOL'SKII, Integral representations of functions, and embedding theorems. (Russian) Nauka, Moscow, 1975. 
5. M. Bownik, Anisotropic Hardy spaces and wavelets. Mem. Amer. Math. Soc. 164(2003), No. 781, 122 pp.

6. M. Bownik, Atomic and molecular decompositions of anisotropic Triebel-Lizorkin spaces. Trans. Amer. Math. Soc., 2005 (to appear).

7. M. Bownik, Atomic and molecular decompositions of anisotropic Besov spaces. Math. Z. 250(2005), No. 3, 539-571.

8. S. Dachkovski, Anisotropic function spaces and related semi-linear hypoelliptic equations. Math. Nachr. 248/249(2003), 40-61.

9. P. Dintelmann, Classes of Fourier multipliers and Besov-Nikolskij spaces. Math. Nachr. 173(1995), 115-130.

10. P. Dintelmann, On Fourier multipliers between anisotropic weighted function spaces. (German) Ph.D. thesis, TH Darmstadt, 1995.

11. D. E. Edmunds and H. Triebel, Function spaces, entropy numbers, differential operators. Cambridge Tracts in Mathematics, 120. Cambridge University Press, Cambridge, 1996.

12. E. W. FARKAS, Anisotropic function spaces, fractals, and spectra of some elliptic and semi-elliptic operators. Ph.D. thesis, Friedrich-Schiller-Universität Jena, Germany, 1998.

13. W. FARKAS, Atomic and subatomic decompositions in anisotropic function spaces. Math. Nachr. 209(2000), 83-113.

14. M. Frazier and B. Jawerth, Decomposition of Besov spaces. Indiana Univ. Math. J. 34(1985), No. 4, 777-799.

15. M. Frazier and B. Jawerth, A discrete transform and decompositions of distribution spaces. J. Funct. Anal. 93(1990), No. 1, 34-170.

16. M. Frazier, B. Jawerth, and G. Weiss, Littlewood-Paley theory and the study of function spaces. CBMS Regional Conference Series in Mathematics, 79. Published for the Conference Board of the Mathematical Sciences, Washington, DC; by the American Mathematical Society, Providence, RI, 1991.

17. G. Garrigós, R. Hochmuth, and A. TABAcCo, Wavelet characterizations for anisotropic Besov spaces with $0<p<1$. Proc. Edinb. Math. Soc. (2) 47(2004), No. $3,573-595$.

18. G. Garrigós and A. TABAcCo, Wavelet decompositions of anisotropic Besov spaces. Math. Nachr. 239/240(2002), 80-102.

19. R. Hоснмuth, Wavelet characterizations for anisotropic Besov spaces. Appl. Comput. Harmon. Anal. 12(2002), No. 2, 179-208.

20. J. Johnsen, Pointwise multiplication of Besov and Triebel-Lizorkin spaces. Math. Nachr. 175(1995), 85-133.

21. G. KyriazIs, Multilevel characterizations of anisotropic function spaces. SIAM J. Math. Anal. 36(2004), No. 2, 441-462 (electronic).

22. G. Kyriazis and P. Petrushev, On the construction of frames for Triebel-Lizorkin and Besov spaces. Proc. Amer. Math. Soc. (to appear).

23. H.-G. LEOPOLD, Boundedness of anisotropic pseudodifferential operators in function spaces of Besov-Hardy-Sobolev type. Z. Anal. Anwendungen 5(1986), 409-417.

24. Y. Meyer, Wavelets and operators. (Translated from the French) Cambridge Studies in Advanced Mathematics, 37. Cambridge University Press, Cambridge, 1992.

25. S. M. Nikol'SkII, Approximation of functions of several variables and embedding theorems. (Russian) Nauka, Moscow, 1977. 
26. Th. Runst and W. Sickel, Sobolev spaces of fractional order, Nemytskij operators, and nonlinear partial differential equations. de Gruyter Series in Nonlinear Analysis and Applications, 3. Walter de Gruyter \& Co., Berlin, 1996.

27. H.-J. Schmeisser and H. Triebel, Topics in Fourier analysis and function spaces. $A$ Wiley-Interscience Publication. John Wiley \& Sons, Ltd., Chichester, 1987.

28. A. Seeger, A note on Triebel-Lizorkin spaces. Approximation and function spaces (Warsaw, 1986), 391-400, Banach Center Publ., 22, PWN, Warsaw, 1989.

29. P. M. SoARDI, On nonisotropic Lipschitz spaces. Harmonic analysis (Cortona, 1982), 115-138, Lecture Notes in Math., 992, Springer, Berlin, 1983.

30. E. M. Stein and S. WAinger, Problems in harmonic analysis related to curvature. Bull. Amer. Math. Soc. 84(1978), No. 6, 1239-1295.

31. B. Stöckert and H. Triebel, Decomposition methods for function spaces of $B_{p, q}^{s}$ type and $F_{p, q}^{s}$ type. Math. Nachr. 89(1979), 247-267.

32. H. Triebel, Fourier analysis and function spaces (selected topics). (Translated from the German) Teubner Verlagsgesellschaft, Leipzig, 1977.

33. H. Triebel, Theory of function spaces. Monographs in Mathematics, 78. Birkhäuser Verlag, Basel, 1983.

34. H. Triebel, Theory of function spaces. II. Monographs in Mathematics, 84. Birkhäuser Verlag, Basel, 1992.

35. H. Triebel, Fractals and spectra. Related to Fourier analysis and function spaces. Monographs in Mathematics, 91. Birkhäuser Verlag, Basel, 1997.

36. H. Triebel, The structure of functions. Monographs in Mathematics, 97. Birkhäuser Verlag, Basel, 2001.

37. H. Triebel, Towards a Gausslet analysis: Gaussian representations of functions. Function spaces, interpolation theory and related topics (Lund, 2000), 425-449, de Gruyter, Berlin, 2002.

38. H. Triebel, Wavelet frames for distributions; local and pointwise regularity. Studia Math. 154(2003), No. 1, 59-88.

39. H. Triebel, Wavelet bases in anisotropic function spaces. Function Spaces, Differential Operators and Nonlinear Analysis. Conference held in Svratka (May, 2004), 370-387, Math. Inst. Acad. Sci. Czech Republic, Praha, 2005.

40. P. Wojtaszczyk, A mathematical introduction to wavelets. London Mathematical Society Student Texts, 37. Cambridge University Press, Cambridge, 1997.

41. M. YAmAZAKI, A quasihomogeneous version of paradifferential operators. I. Boundedness on spaces of Besov type. J. Fac. Sci. Univ. Tokyo Sect. IA Math. 33(1986), No. 1, 131174.

42. M. YAmAZAKI, A quasihomogeneous version of paradifferential operators. II. A symbol calculus. J. Fac. Sci. Univ. Tokyo Sect. IA Math. 33(1986), No. 2, 311-345.

(Received 30.03.2005)

Authors' address:

Mathematical Institute

Friedrich-Schiller-University Jena

D-07740 Jena, Germany

E-mail: haroske@minet.uni-jena.de

tamasi@minet.uni-jena.de 